\title{
Warped Product Semi-Invariant Submanifolds in Almost Paracontact Metric Manifolds
}

\author{
Mehmet AtÇEKen
}

\begin{abstract}
In this paper we have investigated the existence of warped product semi-invariant submanifolds in almost para contact metric manifolds. Finally, we see that there exists no any warped product semiinvariant submanifold in almost para contact metric manifolds such that the contravariant vector field tangent to submanifold.
\end{abstract}

\section{INTRODUCTION}

It is well known that the notations of warped product are widely used in differential geometry as well as physics. The study of warped product manifolds was initiated by R.L. Bishop and B. O'Neill with differential geometric point of view[8]. After then several papers appeared which have dealt with various geometric aspects of warped product submanifolds $[2,5,7,11]$.

The notation of CR-warped product were first introduced by B-Y. Chen. Recently, he studied warped product CR-submanifolds in Kaehler manifolds and shown that there exist no warped product CR-submanifolds in the form $N_{\perp} \times_{f} N_{T}$ in Kaehler manifolds. Therefore he considered warped product CR-submanifolds in the form $N_{T} \times_{f} N_{\perp}$ called CR-warped product by reversing factor manifolds. He established a relationship between the warping function $f$ and the second fundamental form of CR-warped product submanifold in Kaehler manifolds[2, 3].

I. Hasegawa and I. Mihai obtained a similarly inequality for the squared norm of the second fundamental form in terms of the warping function for contact CR-warped products in Sasakian manifolds[9].

In [10], Authors studied the geometry of anti-invariant submanifolds of almost para contact metric manifolds. They obtained a necessary and sufficient condition for a submanifold to be T-invariant.

In this paper we investigate warped products $N=N_{1} \times_{f} N_{2}$ which are warped product semi-invariant submanifolds with respect to case of $\xi$ in

2000 Mathematics Subject Classification. Primary: 53C15; Secondary: 53C42; Tertiary: 53D15.

Key words and phrases. Para Contact Metric Manifold, Warped Product, Warped Product Semi-Invariant Submanifold. 
almost para contact metric manifolds. Our aim in this paper is to study the warped product manifolds in the setting of almost para contact metric manifold.

\section{Preliminaries}

Let $M$ be a $m$-dimensional differentiable manifold, $\varphi, \xi$ and $\eta$ be a tensor field of type $(1,1)$, a contravariant field and a 1 -form on $M$, respectively, satisfying

$$
\begin{aligned}
\varphi^{2} X & =X-\eta(X) \xi, \quad \varphi \xi=0, & & \eta(\varphi X)=0, \quad \eta(\xi)=1 \\
g(\varphi X, \varphi Y) & =g(X, Y)-\eta(X) \eta(Y), & & \eta(X)=g(X, \xi)
\end{aligned}
$$

for any vector fields $X$ and $Y$ on $M$, then $M$ is called almost para contact metric manifold with structure $(\varphi, \xi, \eta, g)[6]$.

By $\bar{\nabla}$ we denote the Levi-Civita connection on almost para contact metri manifold $M$, then the almost para contact metric manifold $M$ is said to be normal if

$$
\left(\bar{\nabla}_{X} \varphi\right) Y=-g(X, Y) \xi-\eta(Y) X+2 \eta(X) \eta(Y) \xi
$$

and

$$
\bar{\nabla}_{X} \xi=\varphi X
$$

for any $X, Y \in \Gamma(T M)[10]$. The covariant derivative of 1 -form $\eta$ is defined by

$$
\left(\bar{\nabla}_{X} \eta\right) Y=X \eta(Y)-\eta\left(\bar{\nabla}_{X} Y\right) .
$$

Thus we have

$$
\left(\bar{\nabla}_{X} \eta\right) Y=g(\varphi X, Y) .
$$

Now let $N$ be an $n$-dimensional immersed submanifold in $M$. Then the Gauss and Weingarten formulas are, respectively, given by

$$
\bar{\nabla}_{X} Y=\nabla_{X} Y+h(X, Y)
$$

and

$$
\bar{\nabla}_{X} V=-A_{V} X+\nabla_{X}^{\perp} Y
$$

for any $X, Y \in \Gamma(T N)$ and $V \in \Gamma\left(T N^{\perp}\right)$, where $\nabla$ and $\nabla^{\perp}$ denote the connections on $N$ and $T N^{\perp}$, respectively, and $A_{V}$ and $h$ are called the shape operator and second fundamental form of $N$ in $M$, respectively[1]. The shape operator $A_{V}$ and the second fundamental for $h$ are related by

$$
g(h(X, Y), V)=g\left(A_{V} X, Y\right)
$$

for any $X, Y \in \Gamma(T N)$ and $V \in \Gamma\left(T N^{\perp}\right)$.

For any vector field $X$ tangent to $N$ we can write

$$
\varphi X=t X+n X
$$


where $t X$ and $n X$ denote tangential and normal components of $\varphi X$, respectively. In the same way, for any vector field $V$ normal to $N$ we put

$$
\varphi V=B V+C V,
$$

where $B V$ and $C V$ are also tangential and normal components of $\varphi V$, respectively.

The submanifold $N$ is said to be invariant if $n$ is identically zero. On the other hand, $N$ is said to be anti-invariant submanifold if $t$ is identically zero.

For submanifolds tangent to the structure vector field $\xi$, there are different classes of submanifolds. Now we will mention the following:

1) A submanifold $N$ tangent to $\xi$ is called an invariant submanifold if $\varphi$ preserves the tangent space of $N$, i.e., $\varphi\left(T_{x} N\right) \subseteq T_{x} N$ for each $x \in N$.

2) A submanifold $N$ tangent to $\xi$ is called an anti-invariant submanifold if $\varphi$ maps the tangent space of $N$ into the normal space, i.e., $\varphi\left(T_{x} N\right) \subset T_{x}^{\perp} N$ for each $x \in N$.

3) A submanifold $N$ tangent to $\xi$ is called semi-invariant submanifold if it admits an invariant distribution $D$ whose orthogonal complementary distribution $D^{\perp}$ is anti-invariant, i.e., $T N=D \oplus D^{\perp} \oplus\{\xi\}$ with $\varphi\left(D_{x}\right) \subseteq D_{x}$ and $\varphi\left(D_{x}^{\perp}\right) \subset T_{x}^{\perp} N$ for each $x \in N$.

Definition 2.1. Let $\left(N_{1}, g_{1}\right)$ and $\left(N_{2}, g_{2}\right)$ be two Riemannian manifolds with Riemannian metrics $g_{1}$ and $g_{2}$, respectively, and $f$ is a positive definite differentiable function on $N_{1}$. The warped product of $N_{1}$ and $N_{2}$ is the Riemannian manifold $N_{1} \times_{f} N_{2}=\left(N_{1} \times N_{2}, g\right)$ equipped with the Riemannian metric tensor such that

$$
g(X, Y)=g_{1}\left(\pi_{1 *} X, \pi_{1 *} Y\right)+f^{2}\left(\pi_{1}(x)\right) g_{2}\left(\pi_{2 *} X, \pi_{2 *} Y\right)
$$

for any $X, Y \in \Gamma(T N)$, where $\pi_{1}$ and $\pi_{2}$ are the canonical projections of $N_{1} \times N_{2}$ onto $N_{1}$ and $N_{2}$, respectively, and $*$ is the symbol for the tangent map. Thus we have $g=g_{1}+f^{2} g_{2}$, where $f$ is called warping function of the warped product. The warped product manifold $N=N_{1} \times_{f} N_{2}$ is characterized by $N_{1}$ and $N_{2}$ are totally geodesic and totally umbilical submanifolds of $N$, respectively[4].

Now we recall the following lemma from [4] for later use.

Lemma 2.1. Let $N=N_{1} \times_{f} N_{2}$ be a warped product manifold with warped function $f$. Then we have

1) $\nabla_{X} Y \in \Gamma\left(T N_{1}\right)$ for any $X, Y \in \Gamma\left(T N_{1}\right)$,

2) $\nabla_{X} U=\nabla_{U} X=X(\ln f) Z$ for any $X \in \Gamma\left(T N_{1}\right)$ and $U \in \Gamma\left(T N_{2}\right)$,

3) $\nabla_{U} W=\nabla_{U}^{N_{2}} W-g(U, W) \frac{1}{f} \operatorname{grad} f$ for any $W, V \in \Gamma\left(T N_{2}\right)$, where $\nabla$ and $\nabla^{N_{2}}$ denote the Levi-Civita connections on $N$ and $N_{2}$, respectively. 
If the manifolds $N_{T}$ and $N_{\perp}$ are invariant and anti-invariant submanifolds of almost para contact metric manifold $M$, then their warped products are in the form 1.) $N_{\perp} \times{ }_{f} N_{T}$ and 2.) $N_{T} \times_{f} N_{\perp}$.

\section{Warped Product Semi-Invariant Submanifolds of An Almost Para Contact Metric Manifold}

Throughout this section we assume that $M$ is an almost para contact metric manifold with structure $(\varphi, \xi, \eta, g)$ and $N=N_{1} \times_{f} N_{2}$ be a warped product semi-invariant submanifold of $M$. Such submanifolds are always tangent to the structure vector field $\xi$. In case $N=N_{\perp} \times_{f} N_{T}$, there are two subcases.

1) $\xi$ is tangent to $N_{T}$,

2) $\xi$ is tangent to $N_{\perp}$.

First we start with $\xi$ is tangent to $N_{T}$.

Theorem 3.1. Let $M$ be an almost para contact metric manifold. Then there exist no warped product semi-invariant submanifolds in the form $N=$ $N_{\perp} \times_{f} N_{T}$ such that $N_{T}$ is an invariant submanifold tangent $\xi$ and $N_{\perp}$ is an anti-invariant submanifold of $M$.

Proof. We suppose that $N=N_{\perp} \times_{f} N_{T}$ is a warped product semi-invariant submanifold of almost para contact metric manifold $M$ such that $N_{T}$ is invariant submanifold tangent to $\xi$ and $N_{\perp}$ is anti-invariant submanifold. Then from Lemma 2.1 we have

$$
\nabla_{X} Z=\nabla_{Z} X=Z(\ln f) X, \quad X \in \Gamma\left(T N_{T}\right), \text { and } Z \in \Gamma\left(T N_{\perp}\right) .
$$

In particular, replacing $X$ by $\xi$ in (10) and by using (3), (5) we obtain

$$
\begin{aligned}
\bar{\nabla}_{Z} \xi & =\nabla_{Z} \xi+h(Z, \xi) \\
\varphi Z & =Z(\ln f) \xi+h(Z, \xi) .
\end{aligned}
$$

The normal and tangential components of (11), respectively, we find

$$
\varphi Z=h(\xi, Z) \text { and } Z(\ln f)=0 \text { for any } Z \in \Gamma\left(T N_{\perp}\right) .
$$

Thus we conclude that $f$ is a constant function on $N_{\perp}$. This completes the proof.

Now we consider $\xi$ is tangent $N_{\perp}$.

Theorem 3.2. Let $M$ be an almost para contact metric manifold. Then there exist no warped product semi-invariant submanifolds in the form $N=$ $N_{\perp} \times_{f} N_{T}$ such that $N_{T}$ is an invariant submanifold and $N_{\perp}$ is an antiinvariant submanifold tangent to $\xi$ of $M$. 
Proof. We suppose that $N=N_{\perp} \times_{f} N_{T}$ is warped product semi-invariant submanifold such that $N_{\perp}$ is an anti-invariant submanifold tangent to $\xi$ and $N_{T}$ is an invariant submanifold of $M$. Then we have

$$
\nabla_{X} Z=\nabla_{Z} X=Z(\ln f) X \text { for any } Z \in \Gamma\left(T N_{\perp}\right), X \in \Gamma\left(T N_{T}\right) .
$$

In particular, replacing $Z$ by $\xi$ and by using (3), (5) we get

$$
\begin{aligned}
\bar{\nabla}_{X} \xi & =\nabla_{X} \xi+h(X, \xi) \\
\varphi X & =\xi(\ln f) X+h(X, \xi) .
\end{aligned}
$$

The tangential components of (13) we conclude that

$$
\varphi X=\xi(\ln f) X
$$

for any $X \in \Gamma\left(T N_{T}\right)$. Applying $\varphi$ to (14) again and consider $\xi \in \Gamma\left(T N_{\perp}\right)$, we get $[\xi(\ln f)]^{2}=1$. This means that $\xi(f)=f$ or $\xi(f)=-f$. Since $N=$ $N_{\perp} \times{ }_{f} N_{T}$ is a warped product, we have $\nabla_{X} \xi=\nabla_{\xi} X=(\xi \ln f) X=\varphi \xi=0$, this is impossible. This proves our result.

Next we research the existence of warped product semi-invariant submanifolds in the form $N=N_{T} \times_{f} N_{\perp}$ in almost para contact metric manifolds. Here there are two subcases such as

1) $\xi$ is tangent to $N_{T}$,

2) $\xi$ is tangent to $N_{\perp}$.

We start with case 1 ).

Theorem 3.3. Let $M$ be an almost para contact metric manifold. Then there exist no warped product semi-invariant submanifolds in the form $N=$ $N_{T} \times_{f} N_{\perp}$ such that $N_{T}$ is invariant submanifold tangent to $\xi$ and $N_{\perp}$ is anti-invariant submanifold of $M$.

Proof. We suppose that $N=N_{T} \times{ }_{f} N_{\perp}$ be a warped product semi-invariant submanifold such that $N_{T}$ is an invariant submanifold tangent to $\xi$ and $N_{\perp}$ is an anti-invariant submanifold of $M$. Then taking account that Lemma 2.1 and by using (3), (5) we have

$$
\varphi Z=\xi(\ln f) Z+h(Z, \xi)
$$

for any $Z \in \Gamma\left(T N_{\perp}\right)$. From the normal and tangential components of (15) we have

$$
\varphi Z=h(Z, \xi) \text { and } \xi(\ln f) Z=0 .
$$

Making use of (7) and (16) we arrive at

$$
g(\varphi Z, \varphi Z)=g(h(Z, \xi), \varphi Z)=g\left(A_{\varphi Z} \xi, Z\right) .
$$


On the other, taking account of $\varphi$ being symmetric and using (2), (5) and Lemma 2.1, we infer

$$
\begin{aligned}
g(\varphi Z, \varphi Z) & =g(\varphi h(Z, \xi), Z)=g\left(\varphi\left(\bar{\nabla}_{\xi} Z-\nabla_{\xi} Z\right), Z\right) \\
& =g\left(\bar{\nabla}_{\xi} \varphi Z-\left(\bar{\nabla}_{\xi} \varphi\right) Z-\varphi(\xi(\ln f) Z), Z\right) \\
& =g\left(\bar{\nabla}_{\xi} \varphi Z, Z\right)-g(-g(\xi, Z) \xi-\eta(Z) \xi+2 \eta(\xi) \eta(Z) \xi, Z) \\
& =-g\left(A_{\varphi Z} \xi, Z\right) .
\end{aligned}
$$

From (17) and (18) we conclude that $\varphi Z=0$. Since $N_{\perp} \neq 0$ and it is anti-invariant, this is a contradiction which proves our assertion.

Now we consider the subcase 2).

Theorem 3.4. Let $M$ be an almost para contact metric manifold. Then there exist no warped product semi-invariant submanifolds in the form $N=$ $N_{T} \times{ }_{f} N_{\perp}$ such that $N_{T}$ is invariant submanifold and $N_{\perp}$ is anti-invariant submanifold tangent to $\xi$ of $M$.

Proof. By using (3) and (5) and consider Lemma 2.1 we get

$$
\varphi X=X(\ln f) \xi+h(X, \xi)
$$

for any $X \in \Gamma\left(T N_{T}\right)$. Since $N_{T}$ is an invariant submanifold and $\xi \in \Gamma\left(T N_{\perp}\right)$ we have

$$
\varphi X=X(\ln f) \xi \text { and } h(X, \xi)=0 .
$$

From the tangential components of (19) we get

$$
X(\ln f)=g(\varphi X, \xi)=\eta(\varphi X)=0 .
$$

This means that the warping function $f$ is constant on $N_{T}$. Thus the proof is complete.

Acknowledgements: I would like to express my gratitude to the referee(s) for valuable comments and suggestions.

\section{REFERENCES}

[1] A. Bejancu, Geometry of CR-Submanifolds, Kluwer, Dortrecht (1986).

[2] B.Y. Chen, Geometry of Warped Product CR-Submanifolds in Kaehler Manifold, Monatsh. Math. 133(2001), 177-195.

[3] B.Y. Chen, Geometry of Warped Product CR-Submanifolds in Kaehler Manifolds II, Monatsh. Math. 134(2001), 103-119.

[4] B. O'Neill, Semi-Riemannian Geometry, Academic Press, New York, 1983.

[5] C. Murathan, K. Arslan, R. Ezentas and I. Mihai, Contact CR-Warped Product Submanifolds in Kenmotsu Space Forms, J. Korean Math. Soc. 42(2005), 1101-1110.

[6] D.E. Blair, Contact Manifolds in Riemannian Geometry, Lecture Notes in Mathematics, Springer-Verlag, New York, Vol. 509, 1976. 
[7] K.A. Khan, V.A. Khan and Sirajuddin, Warped Product Contact CR-Submanifolds of Trans-Sasakian Manifolds, Filomat 21:2(2007), 55-62.

[8] R.L. Bishop and B. O'Neill, Manifolds of Negative Curvature, Trans Amer. Math. Soc. 145(1969), 1-49.

[9] I. Hasegawa and I. Mihai, Contact CR-warped product submanifolds in Sasakian Manifolds, Geom. Dedicata 102(2003), 143-150.

[10] H.B. Pandey and A. Kumar, Anti-Invariant Submanifolds of Almost Paracontact Manifolds. Indian J. pure appl. Math., 16(6)(1985), 586-590.

[11] J. Nikic, Conditions For Invariant Submanifold of a Manifold with the $(\varphi, \xi, \eta, G)$ Structure, Kragujevac J. Math. 25(2003), 147-154.

\author{
Mehmet AtÇeken \\ Gaziosmanpasa University \\ Faculty of Arts and Sciences \\ Department of Mathematics \\ 60100 TOKAT \\ TURKEY \\ E-mail address: matceken@gop.edu.tr
}

